\title{
On an illusion of visual temporal order
}

\author{
MARK S. MAYZNER \\ Loyola University, Chicago, Illinois 60626 \\ and \\ WILLIAM W. AGRESTI \\ University of Michigan, Dearborn, Michigan 48128
}

\begin{abstract}
Earlier work of Sekuler and his associates on visual temporal order is replicated, with a similar experimental paradigm. Our results, as those of Sekuler, suggest an internal mechanism that scans visual inputs in a left-to-right order, but also suggest that such internal scans may also proceed in top-to-bottom or bottom-to-top orders.
\end{abstract}

The starting point for the present study is the report by Sekuler, Tynan, and Levinson (1973) of a series of experiments dealing with visual temporal order. In those experiments, two stimuli were presented in rapid succession side by side on a cathode-ray tube (CRT) display. Subjects responded that the left stimulus occurred first, even when that was not the case. Under several different conditions, this effect of temporal order was tested by presenting the pair of characters in left-to-right order in some of the trials, and right-to-left order in others. The illusion persisted, even under forced-choice testing: The left-to-right order was more frequently reported and more often correctly reported than the right-to-left order.

Because the stimuli were presented next to each other, possible explanations are encouraged that are based on the horizontal nature of the input. Two of the potential explanations were eliminated by other experiments of Sekuler et al. (1973). One concerned the position of the stimuli on the screen as it affected the hemisphere to which the stimuli project. The second, also discounted, would have attributed the illusion to a response bias that left precedes right.

A remaining plausible explanation is that the illusion may be the product of a left-to-right scanning mechanism. Heron (1957) has proposed that such a mechanism operates by jumping to the left end of a list of stimuli and then scanning rightward.

In the present study, we seek another confirmation of the left-to-right illusion of visual temporal order. In addition, we include a separate treatment in which the stimuli are above and below the fixation point. As in the earlier work, the order of presentation varies: either top first then bottom, or bottom first then top. This inclusion of vertical stimuli removes some of the confounding effects that horizontal stimuli present, in terms of visual fields and hemispheres of the brain. The

This research was supported in part by Grants BG22785 and BNS75-09800 A02 to M. S. Mayzner. results from the vertically arranged input may be useful in assessing the proposal that a readout mechanism operates on temporal iconic store.

\section{METHOD}

Two females and six males volunteered to participate in the experiment. The stimuli were presented on a VR-14 CRT display driven by a PDP-8/e computer. The CRT display surface was coated with an ultrashort-persistence (P24) phosphor. The laboratory is described in detail elsewhere (Mayzner, 1968, 1975).

In Treatment A, two letter Xs were presented next to each other $3 \mathrm{~cm}$ apart and centered on the screen. The letters were presented sequentially in two orderings: one left to right, the other right to left. In Treatment $\mathrm{B}$, the two Xs appeared one above the other $3 \mathrm{~cm}$ apart and centered on the display surface. Again, two orderings were used: top to bottom and bottom to top.

The subject, with nonpreferred eye occluded, was seated $2 \mathrm{ft}$ from the CRT display. Four individual-letter presentation times (on-times) were used: 10, 30, 50, and $70 \mathrm{msec}$. The presentation of the second letter in each pair began immediately after the termination of the first; that is, the off-time between inputs was 0 msec. Each subject received both Treatments $A$ and $B$, but the order of the treatments was alternated. Subject 1 received treatments in the order $\mathrm{AB}$, Subject 2 in the order BA, and so on.

The subject was asked to report which letter appeared first in the pair: left or right for Treatment A, top or bottom for Treatment B. For each time-order combination, there were 24 replications. Within each treatment, the presentations were randomized over the four on-times and two orders. Each subject received $2 \times 4 \times 24=192$ displays/treatment, or 384 displays in total.

\section{RESULTS AND DISCUSSION}

Table 1 gives the percentage of letter Xs correctly

Table 1

Mean Percentage Correct Responses, Treatment A

\begin{tabular}{ccccc}
\hline & \multicolumn{4}{c}{ On-Time (in Milliseconds) } \\
\cline { 2 - 5 } Ordering & 10 & 30 & 50 & 70 \\
\hline Left to Right & 54.7 & 66.7 & 84.9 & 95.3 \\
Right to Left & 46.4 & 62.5 & 79.7 & 84.9 \\
\hline
\end{tabular}


reported for each time-order combination in Treatment A. As expected, an analysis of variance shows the effect of on-time to be significant $(p<.01)$. The percent correct are consistently higher for the left-to-right ordering, although this difference is only marginally significant $(.05<\mathrm{p}<.10)$.

The results for Treatment B (Table 2) show consistently higher percentages correct for the ordering that displays the bottom letter first, then the top letter. Although this bottom-to-top sequence yields higher scores, the difference does not appear to be significant.

To help determine if more data on each subject would change the results, two subjects repeated the experiment. Six trials on each of the two subjects produced the results in Table 3 . Each entry in Table 3 gives the percent correct for 288 displays at the listed time-ordering combination.

Of the entries in Table 1, the first column is most comparable to the results of Sekuler et al. (1973). The on-times in both cases are $10 \mathrm{msec}$, with 0 -msec offtimes. The evidence here for the illusion of visual temporal order is not nearly so strong as in the earlier study. The percent correct for left-to-right sequences is $54.7 \%$ vs. $46.4 \%$ for right-to-left sequences. In the Sekuler et al. study, the comparable figures are $60 \%$ vs. $30 \%, 69 \%$ vs. $33 \%$, and $62 \%$ vs. $39 \%$ for different variations of the experiment.

The remainder of Table 1 demonstrates that the higher percentage for the left-to-right order continues as the on-time increases. It would seem that the difference between the two orders should lessen as the on-time increases, because, for very long on-times, say $500 \mathrm{msec}$, both percentages would be $100 \%$ and, therefore, the difference would be zero. Apparently, this lessening in difference occurs after $70 \mathrm{msec}$, because at $70 \mathrm{msec}$,

Table 2

Mean Percentage Correct Responses, Treatment B

\begin{tabular}{ccccc}
\hline & \multicolumn{4}{c}{ On-Time (in Milliseconds) } \\
\cline { 2 - 5 } Ordering & 10 & 30 & 50 & 70 \\
\hline Top to Bottom & 54.2 & 64.1 & 71.4 & 83.9 \\
Bottom to Top & 60.4 & 66.7 & 82.8 & 87.0 \\
\hline
\end{tabular}

Table 3

Mean Percentage Correct Responses, Treatment A, for Two Subjects with Six Trials Each

\begin{tabular}{ccccc}
\hline & \multicolumn{4}{c}{ On-Time (in Milliseconds) } \\
\cline { 2 - 5 } Ordering & 10 & 30 & 50 & 70 \\
\hline Left to Right & 74.3 & 80.2 & 91.3 & 95.8 \\
Right to Left & 39.2 & 53.5 & 79.2 & 89.2 \\
\hline
\end{tabular}

the preference for the left-to-right ordering is actually stronger than at shorter on-times.

The higher scores for the bottom-to-top order in Table 2 might be due to a tendency for the subject's focus to drop siightly from the fixation point. (This comment represents a speculation by the experimenter and was not mentioned by any of the subjects.) Such a slight shift in fixation might encourage reports of observing the bottom $\mathrm{X}$ first.

With more trials per subject, the results (from Table 3) are nearer to those of Sekuler et al. (1973). At $10 \mathrm{msec}$, the percentages correct of $74 \%$ for the left-to-right order and 39\% for the right-to-left order are more in line with the results given above from the earlier study. Also, differences between the scores for the two orderings steadily decrease $(35.1 \%, 26.7 \%, 12.1 \%$, $6.6 \%$ ), as discussed above.

The evidence for the illusion of visual temporal order is much stronger in Table 3 than in Table 1. Recall that several trials with only two subjects produced the Table 3 results. The raw data that led to Table 1 contain considerable individual differences among subjects, especially at short presentation times. At $10 \mathrm{msec}$, for example, three of the eight subjects actually had a higher percent correct for the right-to-left sequences. For example, one subject (M.S.) correctly reported only 6 of 24 left-to-right sequences, but 17 of 24 right-to-left sequences. The two subjects who took the experiment six times had one such reversal each. Also, their scores show no practice effects over the six trials.

In conclusion, the more frequent reporting of left-toright sequences replicated the result of Sekuler et al. (1973) and persisted when the presentation time was increased. The effect was not as strong as in the earlier study, a result that might be attributable to individual differences among subjects. When more extensive data were obtained on two subjects, the illusion of visual temporal order was comparable with that recorded by Sekuler et al.

\section{REFERENCES}

Heron, W. Perception as a function of retinal focus and attention, American Journal of Psychology, 1957, 70, 38-48.

MAYZNER, M. S. The research potential of a computer-based cathode ray tube display system. Behavior Research Methods \& Instrumentation, 1968, 1, 41-43.

MAYZNER, M. S. Studies in visual information processing in man. In $\mathrm{R}$. Solso (Ed.), Information processing and cognition. Hillsdale, N.J: Lawrence Erlbaum, 1975.

Sekuler, R., Tynan, P., \& Levinson, E. Visual temporal order: A new illusiọn. Science, 1973, 180, 210-212.

(Received for publication September 6, 1978.) 